\title{
Locking in the incompressible limit for the element-free Galerkin method
}

\author{
Antonio Huerta*, ${ }^{*}$ and Sonia Fernández-Méndez \\ Departament de Matemàtica Aplicada III, E.T.S. de Ingenieros de Caminos, Canales y Puertos, \\ Universitat Politècnica de Catalunya, Jordi Girona 1, E-08034 Barcelona, Spain
}

\begin{abstract}
SUMMARY
Volumetric locking (locking in the incompressible limit) for linear elastic isotropic materials is studied in the context of the element-free Galerkin method. The modal analysis developed here shows that the number of non-physical locking modes is independent of the dilation parameter (support of the interpolation functions). Thus increasing the dilation parameter does not suppress locking. Nevertheless, an increase in the dilation parameter does reduce the energy associated with the non-physical locking modes; thus, in part, it alleviates the locking phenomena. This is shown for linear and quadratic orders of consistency. Moreover, the biquadratic order of consistency, as in finite elements, improves the locking behaviour. Although more locking modes are present in the element-free Galerkin method with quadratic consistency than with standard biquadratic finite elements. Finally, numerical examples are shown to validate the modal analysis. In particular, the conclusions of the modal analysis are also confirmed in an elastoplastic example.
\end{abstract}

KEY WORDS: locking; volumetric locking, element-free Galerkin; meshless; meshfree

\section{INTRODUCTION}

Locking in finite elements has been a major concern since its early developments. It appears because poor numerical interpolation leads to an over-constrained system. This paper studies locking of the element-free Galerkin (EFG) method near the incompressible limit, i.e. the so-called volumetric locking. In particular, its behaviour is compared with standard finite elements, bilinear and biquadratic, which are recalled in Sections 2.2 and 2.3.

Locking of standard finite elements has been extensively studied. It is well known that bilinear finite elements lock in some problems and that biquadratic elements have a better

\footnotetext{
${ }^{*}$ Correspondence to: Antonio Huerta, Departament de Matemàtica Aplicada III, E.T.S. de Ingenieros de Caminos, Canales y Puertos, Universitat Politècnica de Catalunya, Jordi Girona 1, E-08034 Barcelona, Spain

†E-mail: antonio.huerta@upc.es

Contract/grant sponsor: Ministerio de Educación y Cultura; contract/grant number: TAP98-0421

Contract/grant sponsor: CICYT; contract/grant number: 2FD97-1206

Contract/grant sponsor: Commission for Cultural, Educational and Scientific Exchange, U.S.A. and Spain; contract/grant number: 99258 (1999 program)
} 
behaviour [1,2]. Moreover, locking has also been studied for increasing polynomial degrees in the context of an $h p$ adaptive strategy, see Reference [3]. However, locking in meshless methods is still an open topic. Originally, see Reference $[4,5]$, it was claimed that the EFG method did not exhibit volumetric locking. Now it is clear that this is not true. For instance, Dolbow and Belytschko [6] use the numerical inf-sup condition to analyse the EFG method. Moreover, some authors [6-8] claim that increasing the dilation parameter locking phenomena in meshless methods can be suppressed, or at least attenuated. Their argument is based on numerical examples [7,6] or on the heuristic constraint ratio [8] proposed by Hughes [1]. Finally, they introduce new formulations to remedy this problem $[6,8]$.

Here, a modal analysis (an eigenvector and eigenvalue analysis), see Section 3, is performed in order to clarify this issue. That is, the influence of the dilation parameter on the locking behaviour of FFG near the incompressible limit is determined studying the fundamental modes (base of the solution space) and their corresponding energy (eigenvalue). Moreover, the influence of the order of the approximation is also analysed. Finally, standard linear and non-linear tests are performed in order to illustrate and confirm the conclusions of the modal analysis.

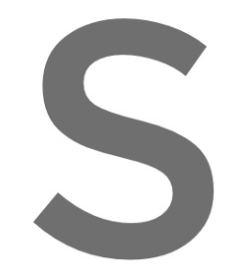

\section{VOLUMETRIC LOCKING IN STANDARD FINITE ELEMENTS}

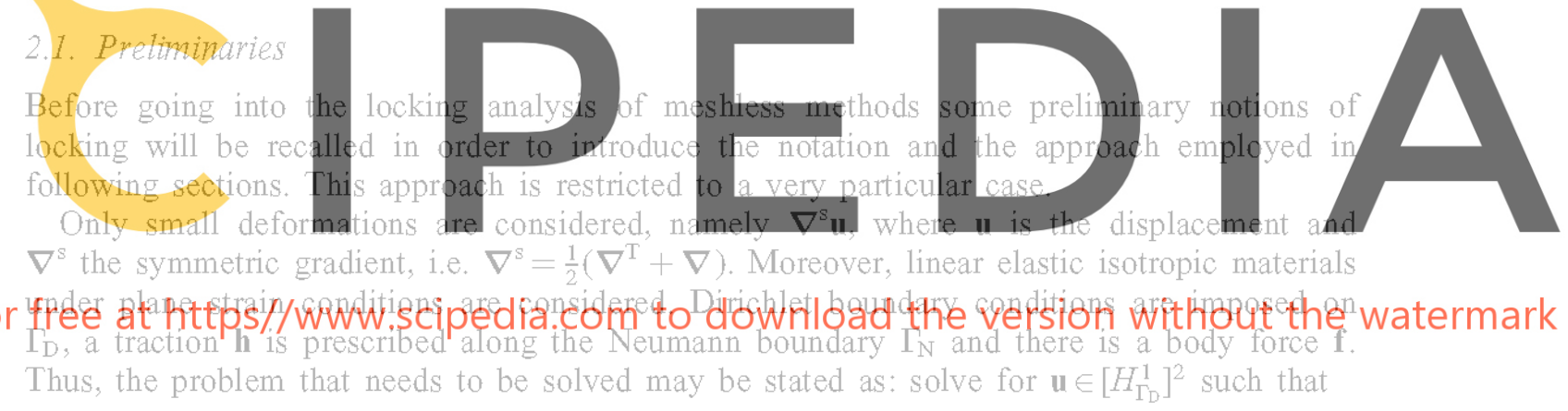

$$
\begin{aligned}
& \frac{E}{1+v} \int_{\Omega} \nabla^{\mathrm{s}} \mathbf{v}: \nabla^{\mathrm{s}} \mathbf{u} \mathrm{d} \Omega+\frac{E v}{(1+v)(1-2 v)} \int_{\Omega}(\nabla \cdot \mathbf{v})(\boldsymbol{\nabla} \cdot \mathbf{u}) \mathrm{d} \Omega \\
& =\int_{\Omega} \mathbf{f} \cdot \mathbf{v} \mathrm{d} \Omega+\int_{\Gamma_{\mathrm{N}}} \mathbf{h} \cdot \mathbf{v} \mathrm{d} \Gamma \quad \forall \mathbf{v} \in\left[H_{0, \Gamma_{\mathrm{D}}}^{1}\right]^{2}
\end{aligned}
$$

In this equation, the standard vector subspaces of $H^{1}$ are employed for the solution $\mathbf{u}$

$$
\left[H_{\Gamma_{\mathrm{D}}}^{1}\right]^{2}:=\left\{\mathbf{u} \in\left[H^{1}\right]^{2} \mid \mathbf{u}=\mathbf{u}_{\mathrm{D}} \text { on } \Gamma_{\mathrm{D}}\right\}
$$

(Dirichlet conditions, $\mathbf{u}_{\mathrm{D}}$, are automatically satisfied) and for the test functions $\mathbf{v}$

$$
\left[H_{0, \Gamma_{\mathrm{D}}}^{1}\right]^{2}:=\left\{\mathbf{v} \in\left[H^{1}\right]^{2} \mid \mathbf{v}=\mathbf{0} \text { on } \Gamma_{\mathrm{D}}\right\}
$$

(zero values are imposed along $\Gamma_{\mathrm{D}}$ ). 
This equation, as discussed in Reference [3], shows the inherent difficulties of the incompressible limit. The standard a priori error estimate emanating from (1) and based on the energy norm, which is induced by the LHS of (1), is

$$
\left\|\mathbf{u}-\mathbf{u}_{h}\right\| \leqslant \inf _{\mathbf{w} \in \mathscr{S}_{h}}\|\mathbf{u}-\mathbf{w}\| \leqslant C_{\mathbf{u}, v, p} h^{f(p)}
$$

where $\mathscr{S}_{h}$ is the finite-dimensional subspace of $\left[H_{\Gamma_{\mathrm{D}}}^{1}\right]^{2}$ in which the approximation $\mathbf{u}_{h}$ is sought, $C_{\mathbf{u}, v, p}$ is a constant independent of $h$ (characteristic size of the mesh), and $f(p)$ is a positive monotone function of $p$ (degree of the polynomials used for the interpolation). The subindices of the constant $C$ indicate that it depends on the Poisson ratio, the order of the interpolation and the exact solution itself.

From (1) one can observe the difficulties of the energy norm to produce a small infimum in (2) for values of $y$ close to 0.5 . In fact, in order to have finite values of the energy norm the divergence-free condition must be enforced in the continuum case, i.e. $\nabla \cdot u=0$ for $u \in\left[H_{\Gamma D}^{1}\right]^{2}$, and also in the finite-dimensional space, i.e. $\nabla \cdot \mathbf{u}_{\hbar}=0$ for $\mathbf{u}_{\hbar} \in \mathscr{S}_{h} \subset\left[H_{\Gamma_{\mathrm{D}}}^{1}\right]^{2}$. In fact, locking will occur when the approximation space $\mathscr{S}_{h}$ is not rich enough for the approximation to verify the divergence-free condition.

Under these conditions, it is evident that locking may be studied from the LHS of (1). This is the basis for the modal analysis of locking. The discrete eigenfunctions (the eigenvectors)

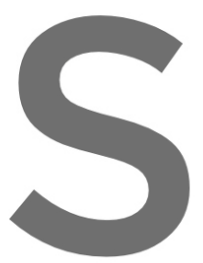
corresponding to the LHS of (1) are computed because they completely describe, in the
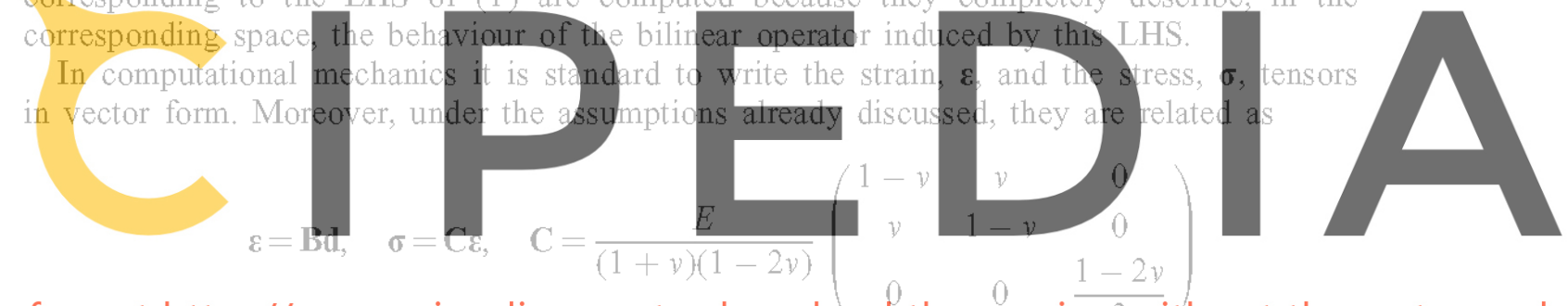

Register for free at https//www.scipedia.com to download the version without the watermark where $d$ is the vector of nodal displacements (the coefficients corresponding to the approximation $\boldsymbol{u}_{h}$ in the base of $\left.\mathscr{S}_{h}\right)$, and $\mathbb{B}$ is the standard matrix relating displacements and strains.

Then, the stiffness matrix can be computed as usual,

$$
\mathbf{K}=\int_{\Omega} \mathbf{B}^{\mathrm{T}} \mathbf{C B} \mathrm{d} \Omega
$$

The modal analysis presented in the following is based on $\mathbf{K}$, which is naturally related to the energy norm in the finite dimensional interpolation space, $\mathscr{S}_{h}$, defined by the finite elements employed (and characterized by $\mathbf{B}$ ).

\subsection{Bilinear finite elements ( $Q 1)$}

The modal analysis of the bilinear (Q1) finite element is performed here. In particular, the incompressible limit is studied by evaluating the eigenvalues associated with each mode as the Poisson ratio, $y$, tends to 0.5 , see Reference [2].

Figures 1-3 present the modes of a bilinear element and the evolution of the eigenvalues as $y$ goes to 0.5 . The logarithm of the eigenvalue is plotted as a function of the logarithm of $0.5-y$. The three rigid body modes have not been plotted because they do not have any 

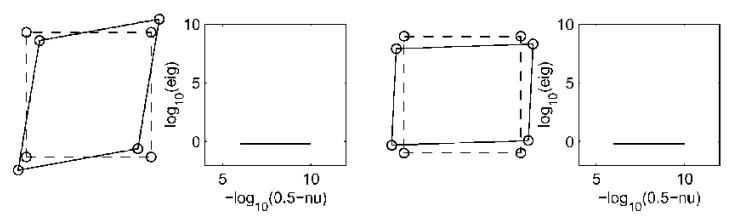

Figure 1. Non-locking modes for one bilinear element (Q1).

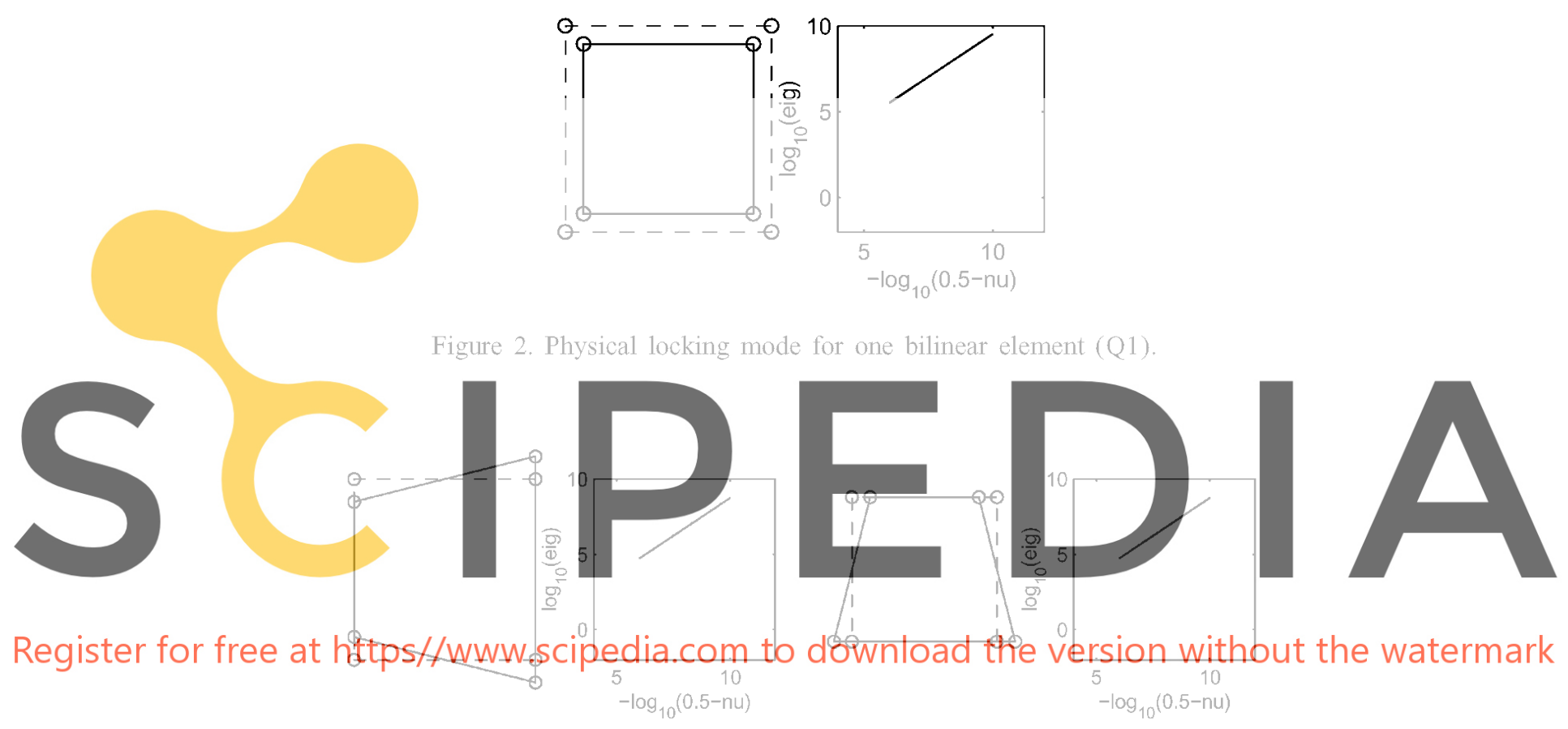

Figure 3. Non-physical locking modes for one bilinear element (Q1).

interest in this study. One can easily recognize in these figures the shear, the stretch, the volumetric and the two hourglass modes. They are classified, as usual, see Reference [2], into three groups: (1) modes that do not present any locking behaviour, (2) modes that do have physical locking - the eigenvalue goes to infinity because it is a volumetric mode-, and (3) modes associated with non-physical locking - in this case, the hourglass modes-.

The first group, Figure 1, presents modes with eigenvalues that do not present an unbounded growth as $v$ goes to 0.5 . In the incompressible limit, $v \simeq 0.5$, the displacement field determined by the eigenvalue can be obtained with a bounded force field. On other hand, the modes that do present locking, see Figures 2 and 3, have eigenvalues that go to infinity when $v$ approaches 0.5 . That is, in the incompressible limit, the force field must be infinite in order to induce the displacements described by the eigenvector. However, such a behaviour is only expected for the volumetric modes and for the others it is non-physical. 

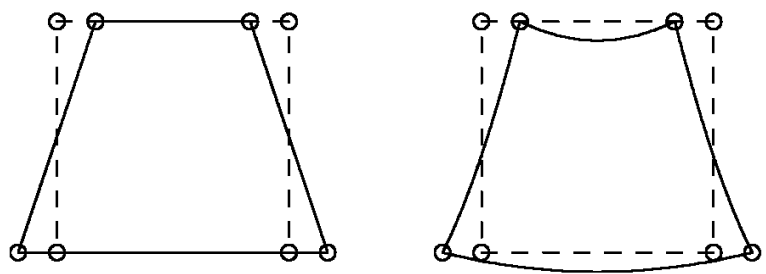

Figure 4. Comparison between the hourglass mode and a divergence-free bending field.

Figure 2 shows the second stretch mode (a volumetric mode), which presents the expected physical locking. The displacement field of this mode does not conserve the area and, consequently, in the incompressible limit it must have an infinite stiffness (infinite eigenvalue). That is, if $v=0.5$ the component of the solution associated with this mode must be zero.

However, the hourglass modes shown in Figure 3 do conserve the total area but suffer from non-physical locking. The interpolation space is not rich enough to ensure the divergence-free condition. In fact the hourglass modes do verify that
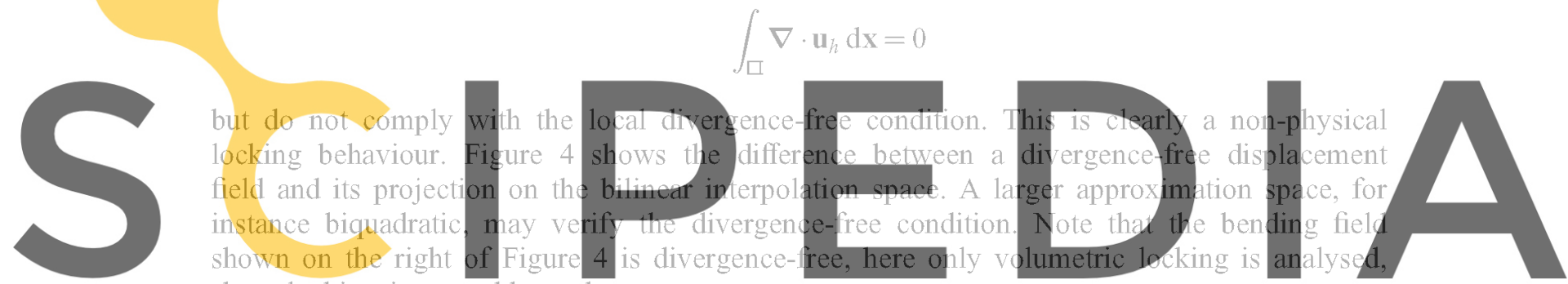
shear locking is not addressed.

In the following section a richer space is chosen: biquadratic finite elements one expects

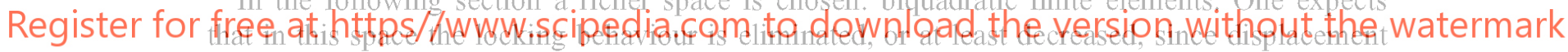
fields closer to a correct one shown in Figure 4 can be obtained.

\subsection{Biquadratic finite elements (Q2)}

Here the modes for the standard biquadratic element are shown. Moreover, this modes are compared with those of the previous bilinear element with an equivalent distribution of nodes. This comparison, standard in finite elements, will be extended to the meshless method.

Figure 5 shows the different modes (the rigid ones are again not shown) for the standard nine-node element. Note that as expected, see Reference [3], there are still some non-physical locking modes. That is, some nodal displacements, which induce global conservation of area, are not able to reproduce a divergence-free field. Nevertheless, most of the standard academic tests [6-8] do perform correctly with the biquadratic element. This simply indicates, and can easily be verified, that the solution field has a zero (or almost zero) coefficient associated with those non-physical locking modes.

Remark 1. In Figure 5, and the following ones, eigenmodes for each group (non-locking, physical locking and non-physical locking) are shown, from left to right and top to bottom, for decreasing magnitudes of the eigenvalue. 
Non-locking
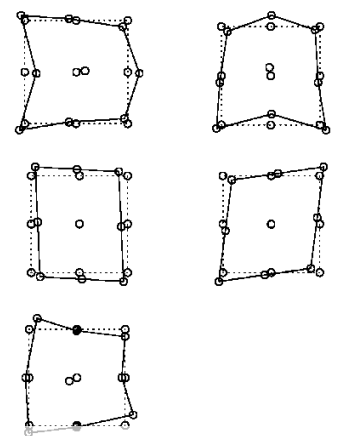

Physical locking
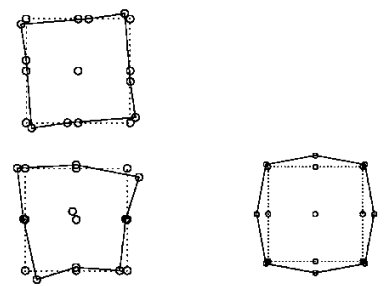
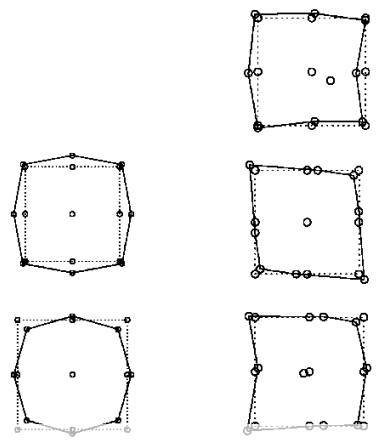

Non-physical locking
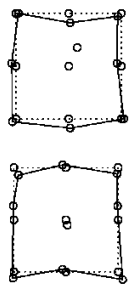

00

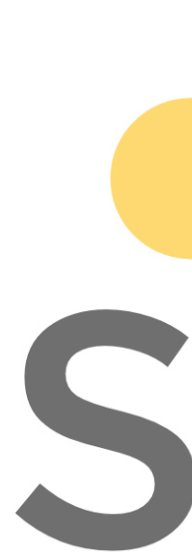

Figure 5. Modes for the Q2 element ( 9 nodes).

\section{Register for free at https//www.scipedia.çom to dowhload the version without the watermark}

\section{Figure 6. Modes of four Q1 elements (9 nodes).}

To further illustrate the importance of the interpolation space the modes of the Q2 element are compared with those of four Q1 elements. Note that both approximation spaces have the same dimension. Nevertheless, from Figures 5 and 6 one can notice that one Q2 element has less non-physical locking modes than the four Q1 elements. Three of the nine non-physical locking modes present in the Q1 elements (see Figure 6) have now, with one Q2, bounded eigenvalues as $v$ goes to 0.5 . Thus a richer space does not necessarily mean a larger space, here both approximation spaces do have the same dimension.

This comparison suggests, however, two important issues that can influence the incompressible limit locking. The first one is related to the bandwidth. For the same number of nodes (the same space dimension) Q2 elements present a larger bandwidth. The second one is the obvious difference between Q1 and Q2 elements: the order of the polynomials in the approximation space. Mesh-free methods allow to study these two phenomena independently; this is the objective of the following section. 
INCOMPRESSIBLE LIMIT FOR GALERKIN METHOD

\section{VOLUMETRIC LOCKING IN ELEMENT-FREE GALERKIN METHODS}

In the previous section two alternative procedures were suggested in order to enrich the interpolation space: (i) increase the bandwidth or (ii) increase the order of the polynomial base.

Mesh-free methods, and in particular the EFG method, are specially suited for independently checking both alternatives: (i) the bandwidth can be increased by simply increasing the dilation parameter, which defines the support of the interpolation functions, keeping the consistency (order of the polynomial approximation) constant and (ii) for large values of the dilation parameter the consistency can be also increased independently.

\subsection{Preliminaries on the element-free Galerkin method}

This section will not be devoted to develop or discuss meshless methods in detail or their relation with moving least-squares (MLS) interpolants. There are well-known references with excellent presentations of meshless methods, see for instance [9-13]. Here some basic notions will be recalled in order to introduce the notation and the approach employed in the following sections.

Meshless methods, or particle methods, are based in a functional interpolation of the form
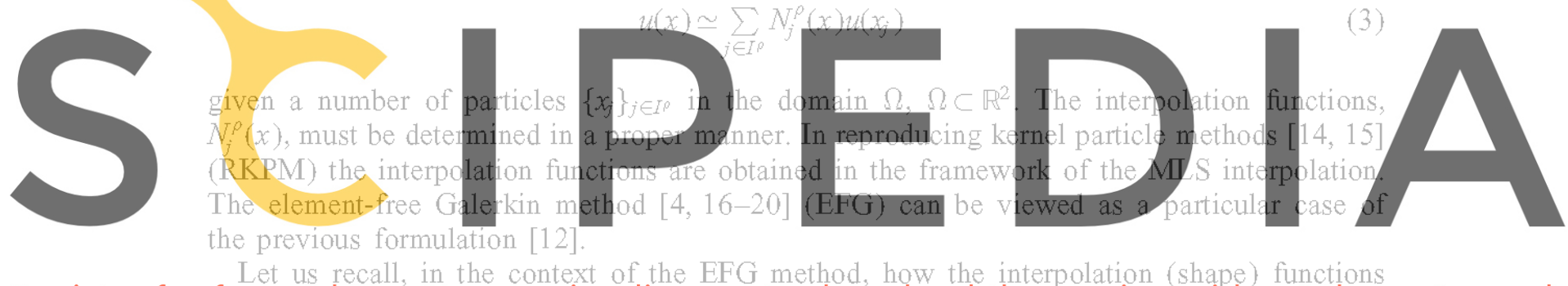

Let us recall, in the context of the EFG method, how the interpolation (shape) functions

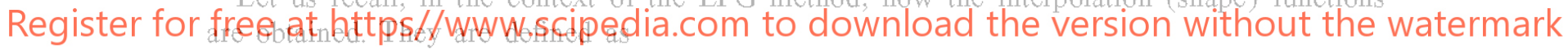

$$
N_{j}^{\rho}(x)=\mathbb{P}^{\mathrm{T}}\left(x_{j}\right) \alpha(x) \phi\left(\frac{x-x_{j}}{\rho}\right)
$$

where the vector $\boldsymbol{\alpha}(x)$ in $\mathbb{R}^{l+1}$ is unknown and $\mathbf{P}(x)=\left\{p_{0}(x), p_{1}(x), \ldots, p_{l}(x)\right\}^{\mathrm{T}}$ is a basis of a polynomial space.

Remark 2 (Interpolation spaces). In one dimension, it is usual that $p_{i}(x)$ coincides with the monomials $x^{i}$, and, in this particular case, $l=m$. For larger spatial dimensions two types of polynomial spaces are usually chosen: the set of polynomials, $\mathscr{P}_{m}$, of total degree $\leqslant m$, and the set of polynomials, $\mathscr{2}_{m}$, of degree $\leqslant m$ in each variable. Both include a complete basis of the subspace of polynomials of degree $m$ (this, in fact, characterizes the a priori convergence rate). Here the second case will be discussed in detail in order to compare with the finite element spaces presented previously, see Sections 2.2 and 2.3. However, two tables summarizing the locking properties are presented for the former case.

The function $\phi(x)$ is a weighting function (positive, even and with compact support) which characterizes the meshless method. For instance, if $\phi(x)$ is continuous together with its first $k$ derivatives, the interpolation is also continuous together with its first $k$ derivatives. In 
RKPM, $\phi(x)$ is directly related to the window function of the reproducing kernel. In (4) the weighting function has been translated, i.e. centred in $x_{j}$, and its support scaled by the dilation parameter $\rho$.

The unknown vector $\boldsymbol{\alpha}(x)$ is determined imposing the so-called reproducibility or consistency condition. It is, in fact, the MLS condition. This reproducibility condition imposes that the interpolation proposed in (3) is exact for polynomials of degree less than or equal to $m$, i.e.

$$
\mathbf{P}(x)=\sum_{j \in I^{\rho}} \mathbf{P}\left(x_{j}\right) N_{j}^{\rho}(x)
$$

For computational purposes, it is usual and preferable $[12,21]$ to centre in $x_{j}$ and scale with $\rho$ also the polynomials involved in previous expressions. Thus, another expression for the shape functions is employed:

$$
N_{j}^{\rho}(x)=\mathbf{P}^{\mathrm{T}}\left(\frac{x-x_{j}}{\rho}\right) \boldsymbol{\alpha}(x) \phi\left(\frac{x-x_{j}}{\rho}\right)
$$

which is similar to (4). The consistency condition becomes in this case:
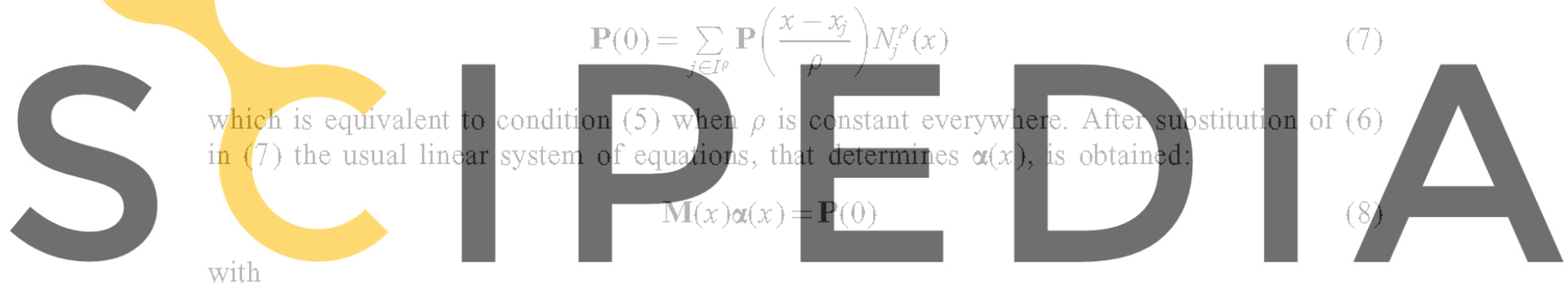

Register for free at https//wwmascipedia.com to dow

Notice that for each $x$ in $\mathbb{R}^{2}$ the previous sum oniy involves those particies whose supportthe support of the weighting function $\phi$-includes $x$. Finally, the approximation that will be used in the weak from of the boundary value problem, viz. (1), is

$$
u(x) \simeq u^{\rho}(x)=\sum_{j \in I^{\rho}} N_{j}^{\rho}(x) u_{j}^{\rho}
$$

where $N_{j}^{\rho}(x)$ are defined by (6), (8) and (9), and $u_{j}^{\rho}$ are the coefficients to be determined.

Remark 3 (Inversibility of $\mathbf{M}$ ). The matrix $\mathbf{M}(x)$ must be regular. In Liu et al. [12] there is a discussion of the necessary conditions. In fact, this matrix can be viewed, see Reference [21], as a Gram matrix defined with a discrete scalar product directly related to the RHS of (9). If this scalar product is degenerated $\mathbf{M}(x)$ is singular. The regularity of $\mathbf{M}(x)$ is ensured by a sufficient amount of particles in the neighbourhood of every point $x$ and located avoiding degenerated patterns, that is,

(i) $\operatorname{card} I_{x}^{p} \geqslant l+1$

(ii) $\nexists F \in\left\langle p_{0}, p_{1}, \ldots, p_{i}\right\rangle \backslash\{0\}$ such that $\forall i \in I_{x}^{P}, F\left(x_{i}\right)=0$. 

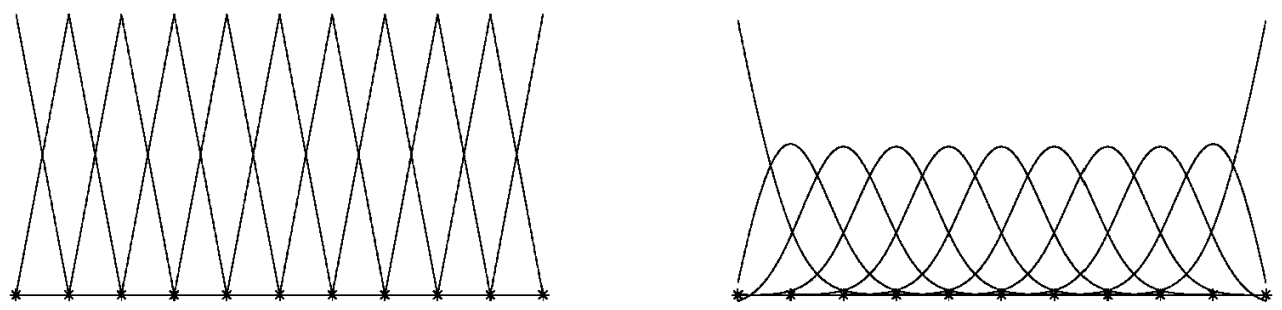

Figure 7. Interpolation functions with $\rho / h \simeq 1$ (similar to finite elements) and $\rho / h=2.6$ for linear consistency, i.e. $\mathbf{P}^{\mathrm{T}}=\{1, x\}$.

where $I_{x}^{\rho}:=\left\{j \in I^{\rho}\right.$ such that $\left.\left|x_{j}-x\right| \leqslant \rho\right\}$. The second condition is easily verified. For instance, for $m=1$ (linear interpolation) the particles cannot lay in the same straight line or plane for, respectively, 2D and 3D. In 1D, for any value of $m$, it suffices that different particles do not have the same position. Under these conditions one can compute the vector $\alpha$ at each point and thus determine the shape functions, $N_{j}^{\rho}(x)$.

Remark 4. The consistency conditions (5) and (7) are equivalent if the dilation parameter $\beta$ is constant. When the dilation parameter varies at each particle another definition of the

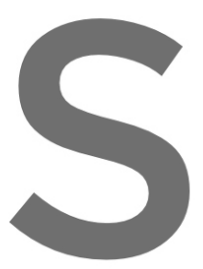
shape functions is recor

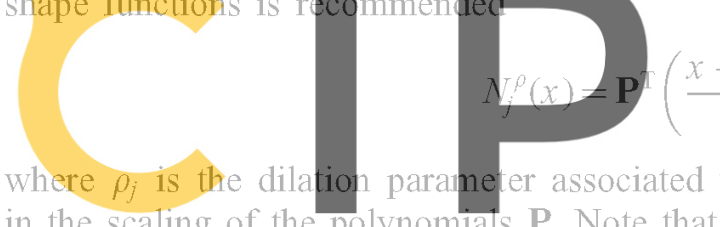

in the scaling of the polynomials $\mathbb{P}$. Note that
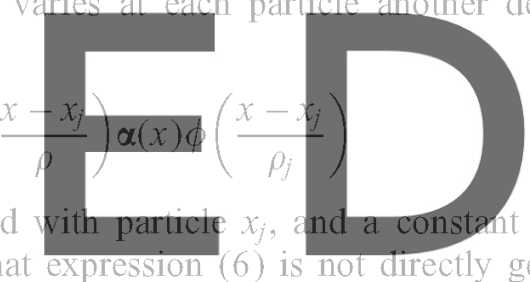

constant value $\rho$ is typically chosen as the mean value of all the $\rho_{j}$. The consistency condition

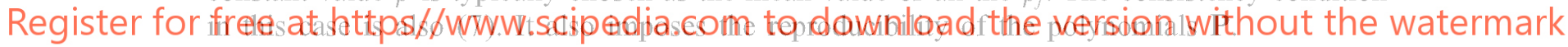

Remark 5. The interpolation is characterized by the order of consistency required, i.e. the basis of polynomials employed $\mathbf{P}$, and by the ratio between the dilation parameter and the particle distance, $\rho / h$. In fact, the bandwidth of the stiffness matrix increases with the ratio $\rho / h$ because the card $I_{x}^{\rho}$ increases (more particles lie inside the circle of radius $\rho$ ), see for instance Figure 7. The influence of these two variables, order of consistency $m$ and ratio $\rho / h$, is studied next.

Remark 6 (Definition of weighting/window function). The weighting function introduced in (4) may be defined in various manners. Here, for simplicity a rectangular support is chosen. Thus, $\phi(\mathbf{x})=\phi_{1 \mathrm{D}}\left(\left|x_{1}\right|\right) \phi_{1 \mathrm{D}}\left(\left|x_{2}\right|\right)$ where $\phi_{1 \mathrm{D}}$ is the cubic spline in one dimension,

$$
\phi_{1 \mathrm{D}}(s)= \begin{cases}\frac{2}{3}+4(s-1) s^{2} & 0 \leqslant s \leqslant 0.5 \\ \frac{4}{3}(1-s)^{3} & 0.5 \leqslant s \leqslant 1 \\ 0 & 1 \leqslant s\end{cases}
$$

However, similar results can be obtained with Gaussian weighting functions or with circular supports, $\phi(\mathbf{x})=\phi_{1 \mathrm{D}}(|\mathbf{x}|)$. 


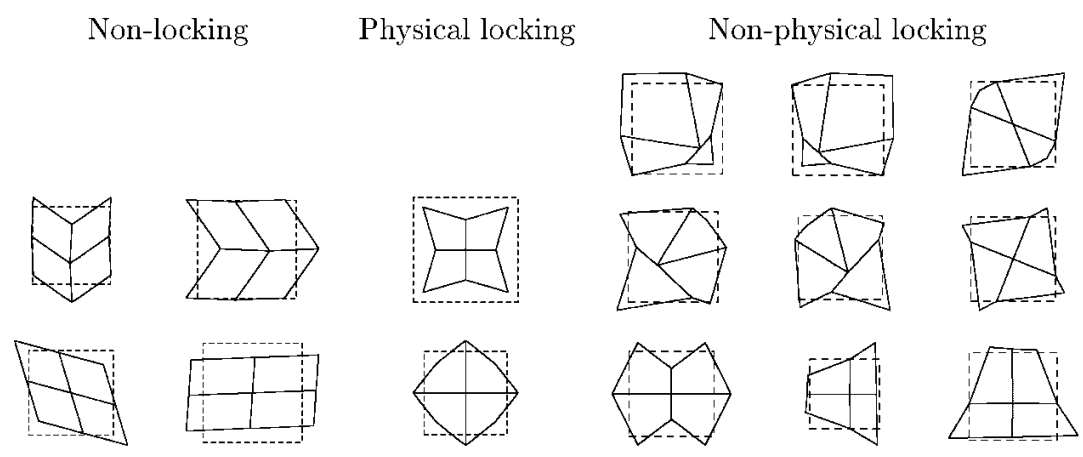

Figure 8. Modes for a $3 \times 3$ distribution of particles with $\mathscr{Q}_{1}$ and $\rho / h=1.2$.

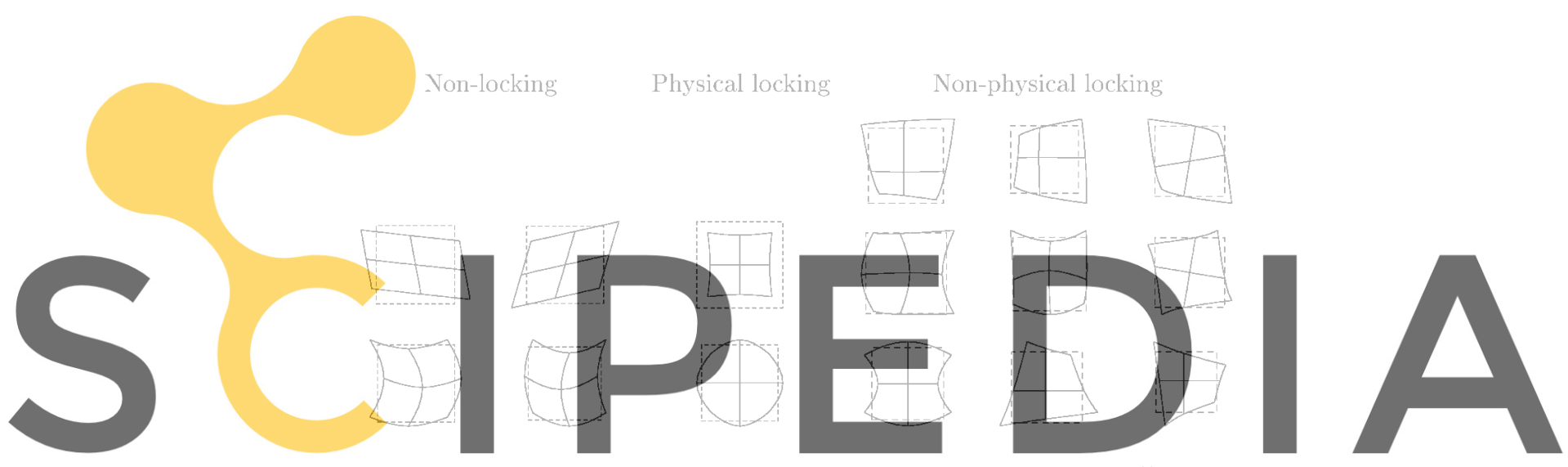

Figure 9. Modes for a $3 \times 3$ distribution of particles with $\mathscr{Q}_{1}$ and $\rho / h=2,2$.

\section{Register for free at https//www.scipedia.com to download the version without the watermark}

\subsection{Locking for bilinear consistency}

In order to compare the results of EFG with the previous ones obtained with finite elements, see Sections 2.2 and 2.3, a distribution of $3 \times 3$ particles is employed. Bilinear consistency corresponds to the polynomial space $\mathscr{Q}_{1}$, namely $\mathbf{P}=\{1, x, y, x y\}^{\mathrm{T}}$. Figures 8 and 9 show the modes already classified for two different dilation parameters, $\rho / h=1.2$ and 2.2 .

As expected the case of $\rho / h=1.2$ does coincide with the Q1 element, see Figure 6. Recall that at the Gauss points the shape functions of the Q1 finite element and the interpolation functions for EFG with $\rho / h=1.2$ are almost identical, thus the eigenvalue analysis should give similar results.

By increasing $\rho / h$ up to 2.2 with the same consistency requirement the bandwidth of the matrix is increased. Nevertheless, the same number of non-physical locking modes is obtained. Notice, however, that an increase of the dilation parameter improves the smoothness of the approximation. The same modes are obtained but the interpolation of the displacements is more smooth. This clearly indicates that an increase of the dilation parameter does not preclude locking. In fact the same modes will still present locking.

This conclusion does not contradict previous numerical experiments [6-8]. In Figure 10 the evolution of eigenvalue as $v$ goes to 0.5 is plotted for the same non-physical locking mode 

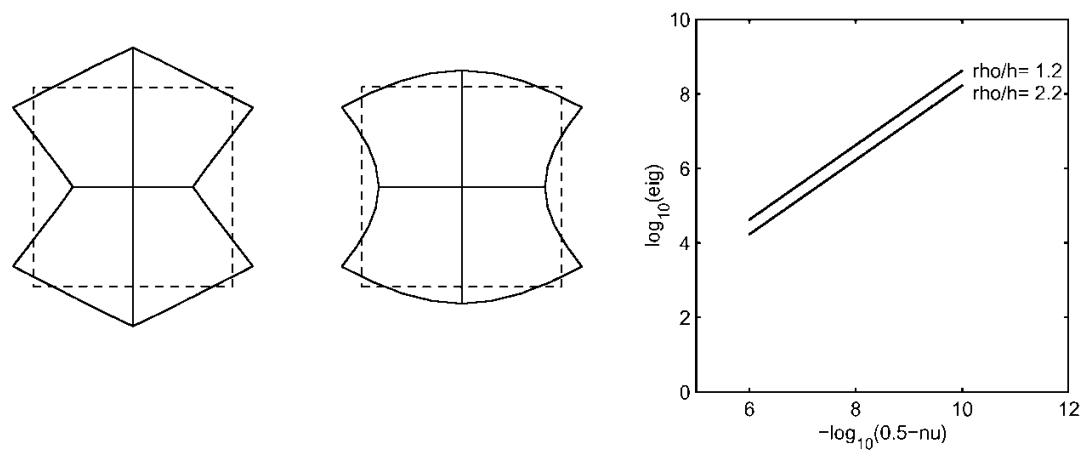

Figure 10. Evolution of the eigenvalue as $v$ goes to 0.5 for the same non-physical locking mode obtained with $\rho / h=1.2$, and 2.2 .

obtained first with $\rho / h=1.2$ and then with 2.2. As noticed before the shape of the mode is improved increasing the dilation parameter, i.e. the interpolation of the displacements is more smooth. This is a clear indication that at a given point $\mathbf{x}$ more particles interact ( $\operatorname{card} I_{x}^{\rho}$ is increased) and a better interpolation is obtained. This better approximation does not preclude locking but reduces its effect by decreasing the eigenvalue. As $v$ goes to 0.5 the energy needed grows unbounded but for a constant $v$ as $\rho / h$ increases the eigenvalue decreases. Thus volumetric locking is not suppressed when the dilation parameter increases but, as noticed in References [6-8] its effect is attenuated.

If the patch of particles is increased, in order to use larger dilation parameters, similar conclusions are drawn:

1. The number of non-physical locking modes is independent of the ratio $\rho / h$.

2. An increase of the dilation parameter decreases the eigenvalue (amount of energy) of the locking mode attenuating, but not suppressing, the volumetric locking (in the incompressible limit the same problems will occur).

Figures 11-13 show the modes for a $4 \times 4$ particle distribution. This patch can be compared with nine Q1 elements. Bilinear consistency is still imposed, $\mathbf{P}=\{1, x, y, x y\}^{\mathrm{T}}$, and the ratios studied correspond to $\rho / h=1.2,2.2$ and 3.2. Table I presents a summary of these results. Moreover, Figure 14 compares the evolution of the eigenvalue associated with the same nonphysical locking mode for the previously indicated ratios.

The same conclusions are obtained with a larger number of particles, Table II presents a summary of the results.

Remark 7. There is a major difference between finite element and EFG interpolation functions: the influence of the boundary on the interpolation functions. This is not important for ratios $\rho / h$ close to 1 , but becomes evident for larger values of $\rho / h$, see Figure 7 . The interpolation functions whose support crosses the boundary differ from those in the interior of the domain. Due to this, the modal analysis performed here, and its comparison with finite elements, is repeated for different distributions of particles. Here, for briefness, results are shown up to $5 \times 5$, see Table II, but as noticed earlier the same conclusions are drawn for larger 


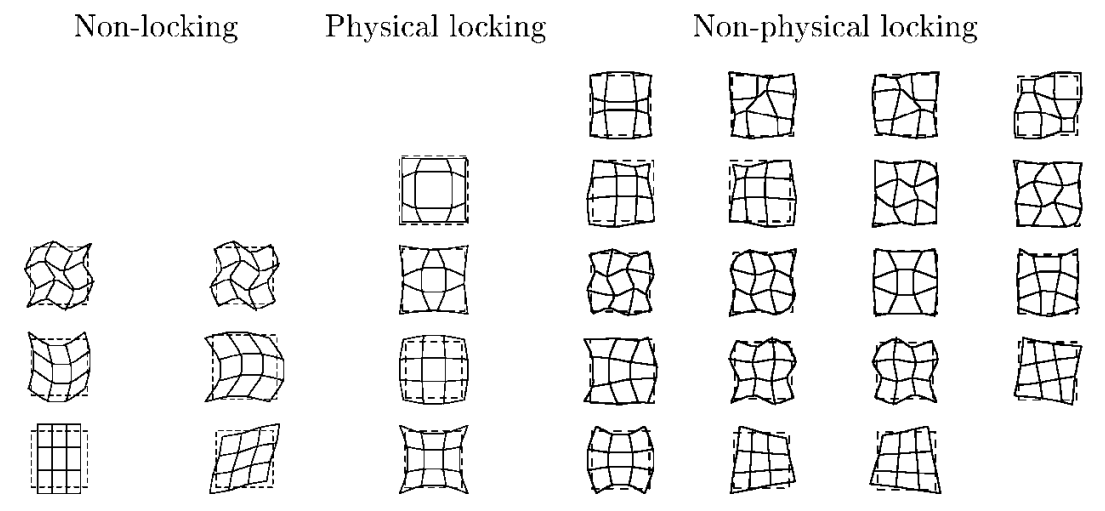

Figure 11 . Modes for a $4 \times 4$ distribution of particles with $\mathscr{Q}_{1}$ and $\rho / h=1.2$.

Non-locking

Physical locking

Non-physical locking

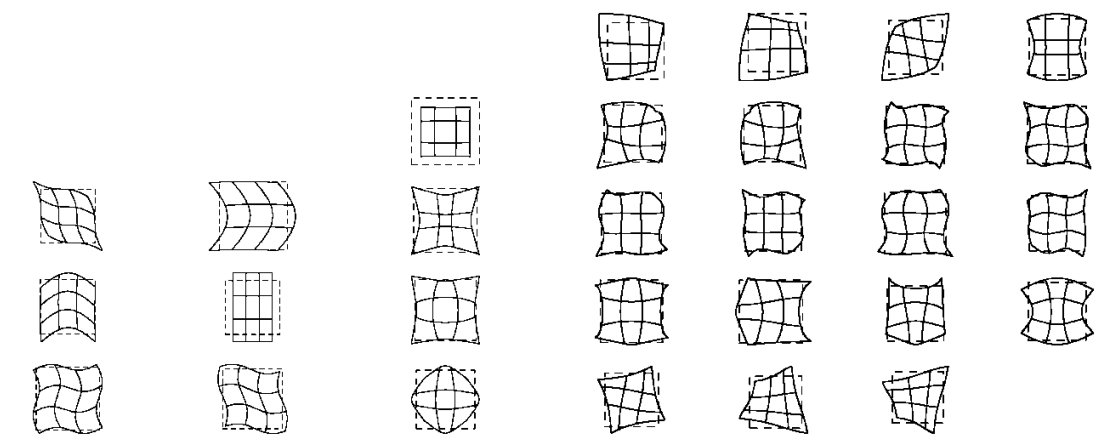

Figure 12. Modes for a $4 \times 4$ distribution of particles with $\mathscr{Q}_{1}$ and $\rho / h=2.2$.

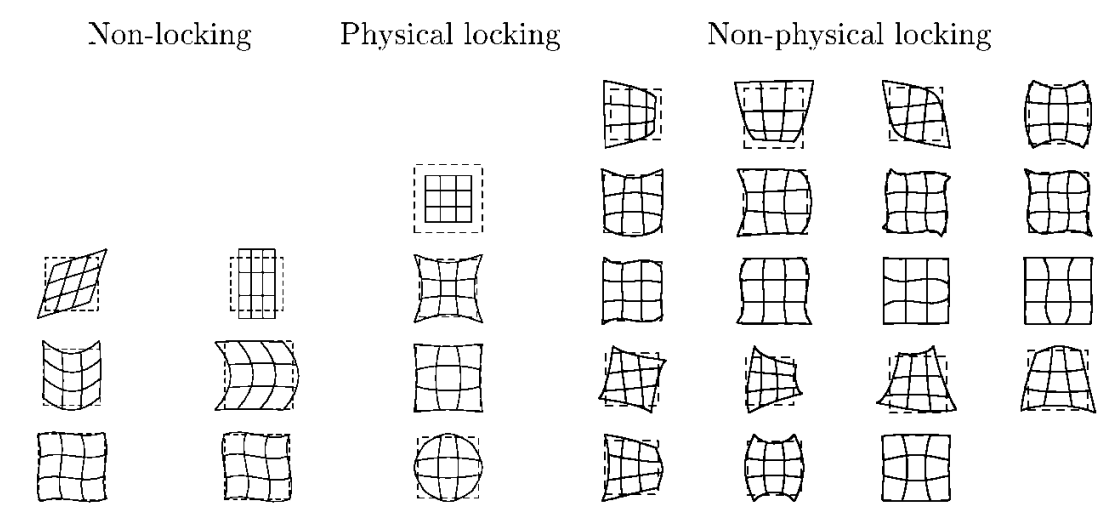

Figure 13. Modes for a $4 \times 4$ distribution of particles with $\mathscr{Q}_{1}$ and $\rho / h=3.2$. 
INCOMPRESSIBLE LIMIT FOR GALERKIN METHOD

Table I. Mode classification for a distribution of $4 \times 4$ particles/nodes.

\begin{tabular}{lccccccc}
\hline & & & $\rho / h=2.2$ & $\rho / h=3.2$ & $\rho / h=2.2$ & $\rho / h=3.2$ & $\rho / h=3.2$ \\
& Q1 & Q2 & $\mathscr{Q}_{1}$ & $\mathscr{Q}_{1}$ & $\mathscr{Q}_{2}$ & $\mathscr{Q}_{2}$ & $\mathscr{2}_{3}$ \\
\hline Non-locking & 6 & - & 6 & 6 & 9 & 9 & 14 \\
Physical locking & 4 & - & 4 & 4 & 4 & 4 & 4 \\
Non-physical locking & 19 & - & 19 & 19 & 16 & 16 & 11 \\
\hline
\end{tabular}
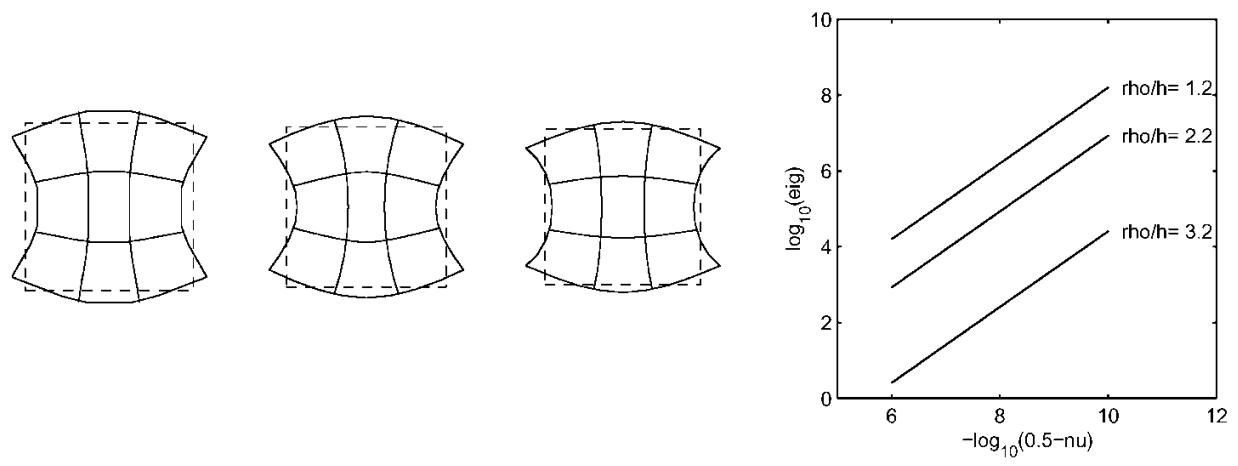

Figure 14. Evolution of the eigenvalue as $v$ goes to 0.5 for the same non-physical locking mode obtained with $\rho / h=1.2,2.2$, and 3.2 .

Table II. Mode classification for a distribution of $5 \times 5$ particles/nodes.

\begin{tabular}{lrrrrrrr}
\hline & & & $\rho / h=2.2$ & $\rho / h=3.2$ & $\rho / h=2.2$ & $\rho / h=3.2$ & $\rho / h=3.2$ \\
& Q1 & Q2 & $\mathscr{Q}_{1}$ & $\mathscr{Q}_{1}$ & $\mathscr{Q}_{2}$ & $\mathscr{Q}_{2}$ & $\mathscr{Q}_{3}$ \\
\hline Non-locking & 8 & $\mathbf{1 6}$ & 8 & 8 & $\mathbf{1 1}$ & $\mathbf{1 1}$ & 16 \\
Physical locking & 6 & 6 & 6 & 6 & 6 & 6 & 6 \\
Non-physical locking & 33 & $\mathbf{2 5}$ & 33 & 33 & $\mathbf{3 0}$ & $\mathbf{3 0}$ & 25 \\
\hline
\end{tabular}

patches of particles (and lower-enough dilation parameters in order to decrease the influence of the boundary interpolation functions).

Remark 8. At this point it seems important to notice that although the modal analysis suggests that increasing the dilation parameter locking is alleviated, in practice the dilation parameter cannot be taken as arbitrarily large. Recall that the EFG method is based on an MLS approximation. The local nature of such approximation will be lost for arbitrarily large $\rho$. In fact, for large-enough values of $\rho$ a global least-squares approach is recovered. The numerical examples shown in Section 4 corroborate this issue.

Remark 9. Non-physical, or physical, locking modes are determined depending on the conservation, or not, of the area. In order to verify the conservation of area and to preclude numerical errors two techniques are used: (1) numerical integration of the divergence of the 
Non-locking
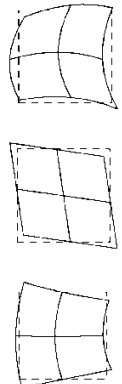
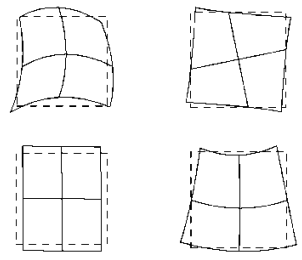

Physical locking

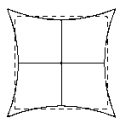

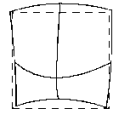

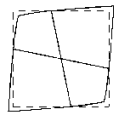

Non-physical locking

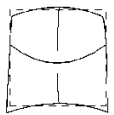

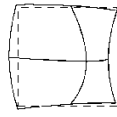
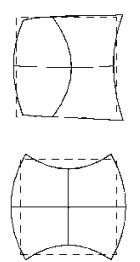

Figure 15 . Modes for a $3 \times 3$ distribution of particles with $\mathscr{Q}_{2}$ and $\rho / h=2.2$.

Non-locking
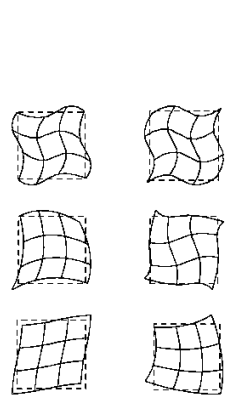

Physical locking
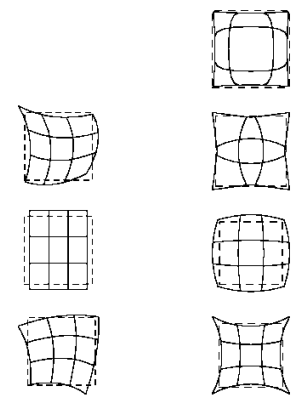
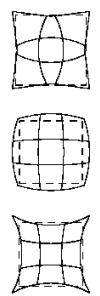

Non-physical locking
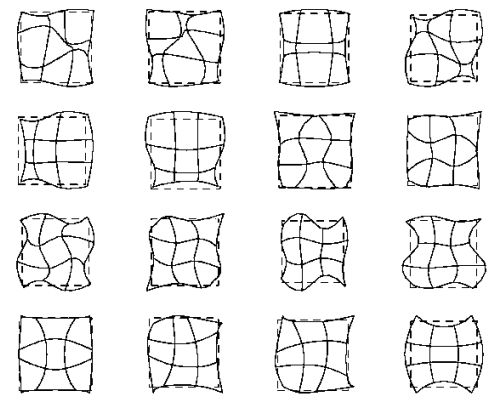

Figure 16. Modes for a $4 \times 4$ distribution of particles with $\mathscr{Q}_{2}$ and $\rho / h=2.2$.

displacement and (2) computation of the displacement flux along the edges. Both methods give the same results with a clear threshold (several orders of magnitude) between constant or variable area.

\subsection{Locking for biquadratic consistency}

In finite elements when the order of consistency is increased volumetric locking is alleviated, and in some cases suppressed [3]. As previously done for finite elements, quadratic consistency is studied. First, the results previously obtained with the Q2 element are reproduced with the EFG method. Thus a domain defined with a distribution of $3 \times 3$ particles is employed with consistency in $\mathscr{2}_{2}$, i.e. $\mathbf{P}=\left\{1, x, y, x y, x^{2}, y^{2}, x^{2} y, x y^{2}, x^{2} y^{2}\right\}^{\mathrm{T}}$. Figure 15 shows the modes in this case, which, as expected, do coincide with the modes of the Q2 element, see Figure 5. The ratio $\rho / h$ is in this case 2.2. Every point in the domain is under the influence of the nine particles. In fact, second order consistency cannot be obtained with $\rho / h=1.2$ because the first necessary condition presented in Remark 3 is not satisfied.

This reduction in the number of non-physical locking modes is also observed when a $4 \times 4$ distribution of particles is used. See Figures 16 and 17 which should compare with 


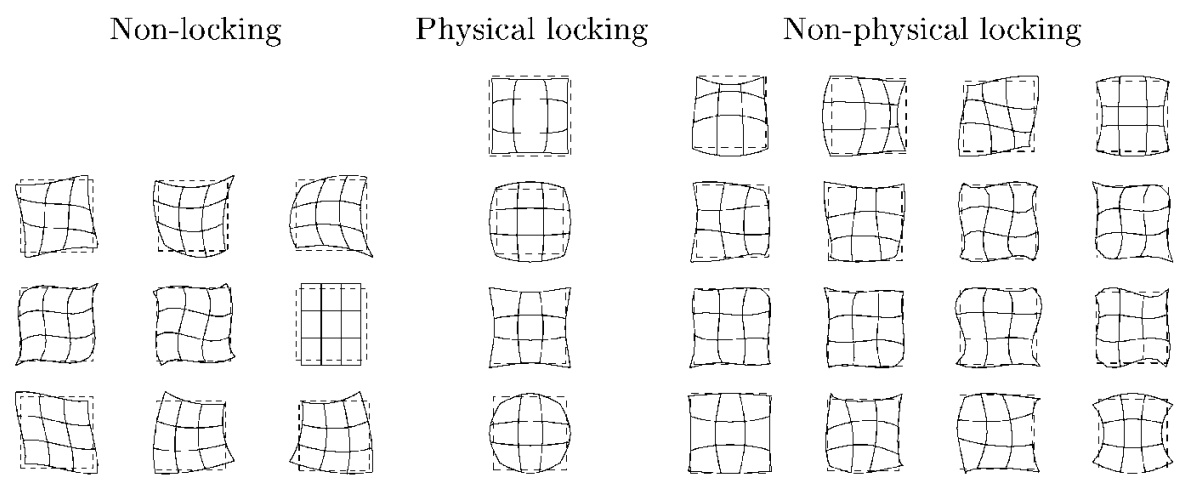

Figure 17 . Modes for a $4 \times 4$ distribution of particles with $\mathscr{Q}_{2}$ and $\rho / h=3.2$.

Table III. Mode classification for a distribution of $4 \times 4$ particles/nodes.

\begin{tabular}{lrrrrr}
\hline & P1 & P2 & $\mathscr{P}_{1}$ & $\mathscr{P}_{2}$ & $\mathscr{P}_{3}$ \\
\hline Non-locking & 11 & - & 6 & 8 & 11 \\
Physical locking & 6 & - & 4 & 4 & 3 \\
Non-physical locking & 12 & - & 19 & 17 & 15 \\
\hline
\end{tabular}

Table IV. Mode classification for a distribution of $5 \times 5$ particles/nodes.

\begin{tabular}{lrrrrrrr}
\hline & P1 & P2 & P4 & $\mathscr{P}_{1}$ & $\mathscr{P}_{2}$ & $\mathscr{P}_{3}$ & $\mathscr{P}_{4}$ \\
\hline Non-locking & 15 & 23 & 27 & 8 & 10 & 13 & 17 \\
Physical locking & 10 & 7 & 6 & 6 & 6 & 5 & 6 \\
Non-physical locking & 22 & 17 & 14 & 33 & 31 & 29 & 24 \\
\hline
\end{tabular}

Figures 11-13. A summary of these results is shown in Table I. This reduction of nonphysical modes due to an increase in the order of consistency is not affected when the dilation parameter is modified. This results cannot be compared with the Q2 element because it is not possible with biquadratic elements to obtain a distribution of $4 \times 4$ nodes.

If a distribution of $5 \times 5$ particles is chosen, results may be compared between EFG, Q1 and Q2 finite elements. The results are presented in Table II. Again, the number of non-physical modes is reduced compared with the linear order of consistency. However, compared with the Q2 element, the EFG method has more non-physical locking modes than the biquadratic finite element. Recall that in EFG all approximation functions are identical. This is not the case for finite elements, midside nodes have different shape functions than corner nodes.

Finally, for completeness, Tables III and IV show, for the same $4 \times 4$ and $5 \times 5$ particle distribution, a comparison between the complete set of polynomials $\mathscr{P}_{m}$ (degree $\leqslant m$ ), see Remark 2, and their corresponding finite elements. Notice that similar conclusions can be drawn. 


\section{NUMERICAL EXAMPLES}

In this section, three standard academic examples are used to illustrate the previously obtained results.

\subsection{The cantilever beam}

A beam with linear isotropic material, under plane strain conditions and with a parabolic traction applied to the free end is considered, as shown in Figure 18. Displacements in both directions are prescribed at $\Gamma_{\mathrm{D}}$. The prescribed displacements and the applied traction are such that the solution is known $[1,6]$ :

$$
\begin{aligned}
u_{x} & =-2 \frac{1-v^{2}}{E} y\left[(48-3 x) x+\left(2+\frac{v}{1-v}\right)\left(y^{2}-0.25\right)\right] \\
u_{y} & =2 \frac{1-v^{2}}{E}\left[3 \frac{v}{1-v} y^{2}(8-x)+\left(4+5 \frac{v}{1-v}\right) \frac{x}{4}+(24-x) x^{2}\right] \\
\sigma_{x x} & =-12 y(8-x), \quad \sigma_{y y}=0, \quad \sigma_{x y}=6\left(0.25-y^{2}\right)
\end{aligned}
$$

The problem is solved with uniform distributions of nodes, when FE are used, or particles, when EFG is used.

Figure 19 shows the relative $L_{2}$ error in displacements for $v=0.3,0.4999$ and 0.499999 . Results are shown for three-noded linear FE (P1), four-noded bilinear FE (Q1), six-noded

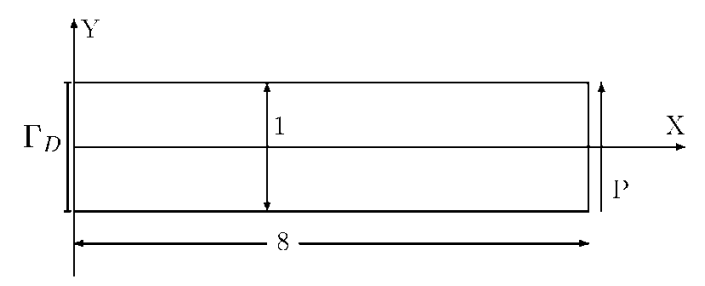

Figure 18. Cantilever beam problem.
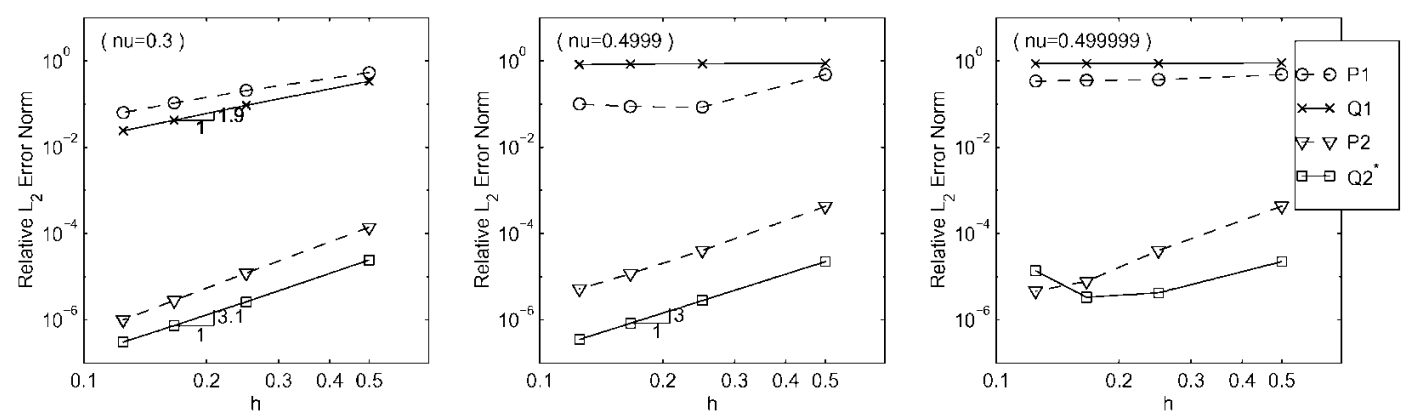

Figure 19. Relative $\mathrm{L}_{2}$ error with $\mathrm{FE}$ and $v=0.3,0.4999,0.499999$. 

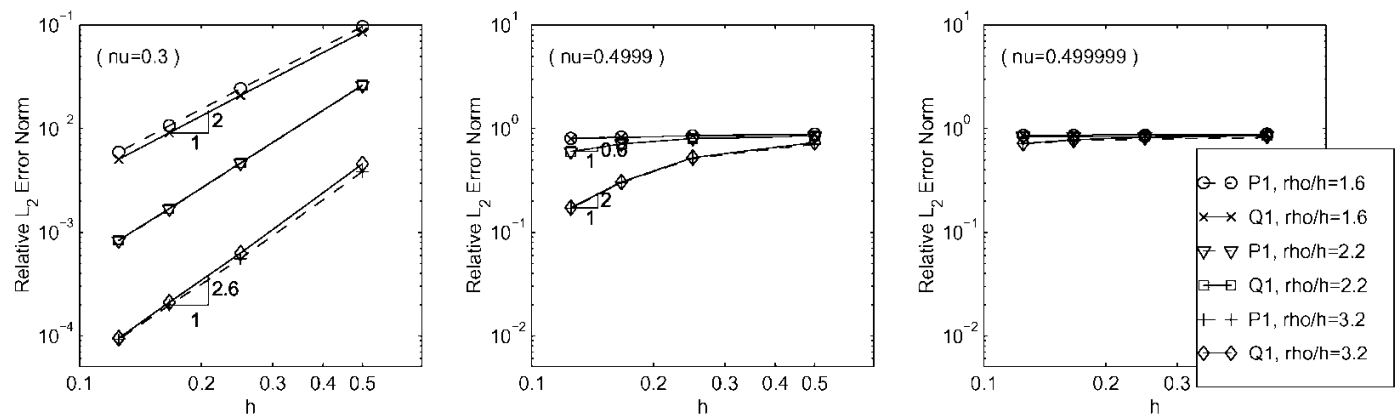

Figure 20. Relative $\mathrm{L}_{2}$ error with $\mathrm{EFG}$ and $v=0.3,0.4999,0.499999$
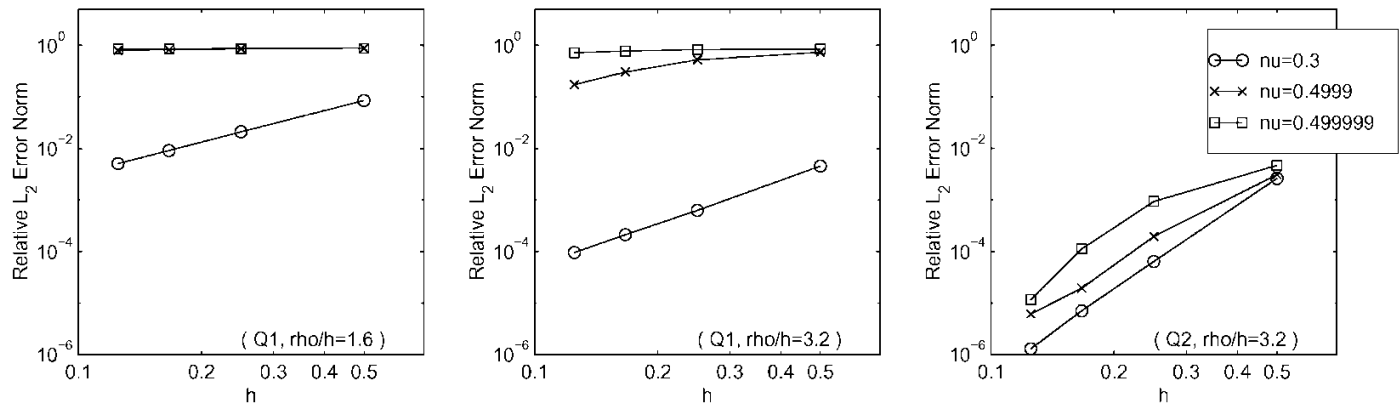

Figure 21. Relative $\mathrm{L}_{2}$ error with $\mathscr{Q}_{1}$ and $\rho / h=1.6$ (left), with $\mathscr{Q}_{1}$ and $\rho / h=3.2$ (centre) and with $\mathscr{Q}_{2}$ and $\rho / h=3.2$ (right).

quadratic FE (P2) and eight-noded FE (Q2*). The typical convergence rates are obtained when $v=0.3$, but, as expected, results degrade as $v$ gets closer to the incompressible limit 0.5. All interpolations suffer from volumetric locking. However, the best results are obtained with interpolations of order two, where locking effects are negligible when $\nu=0.4999$ and good results are still obtained when $v=0.499999$.

Similar results are shown for EFG in Figures 20 and 21. The cubic spline weighting function with circular support is used to define the EFG shape functions and Lagrange multipliers are used in order to impose the prescribed displacements.

Interpolations with consistency of order one, $\mathscr{P}_{1}$ and $\mathscr{Q}_{1}$, are considered in Figure 20 with three different values of the ratio $\rho / h$. Note that, for a given $\rho / h$, similar behaviours are obtained with the $\mathscr{P}_{1}$ and $\mathscr{Q}_{1}$ interpolations, as the modal analysis showed. Far from the incompressible limit, when $v=0.3$, the convergence is as expected or even better. The best solutions are always obtained when $\rho / h=3.2$. However, near the incompressible limit the solution suffers from locking for all values of $\rho / h$ : the solution degrades as $v$ gets closer to the incompressible limit as can be seen in Figure 21. Figure 21 also shows the results obtained with a $\mathscr{Q}_{2}$ interpolation. In this example, with consistency imposed in $\mathscr{Q}_{2}$, locking effects are small since the solution has an almost zero projection in the subspace of non-physical locking modes. 

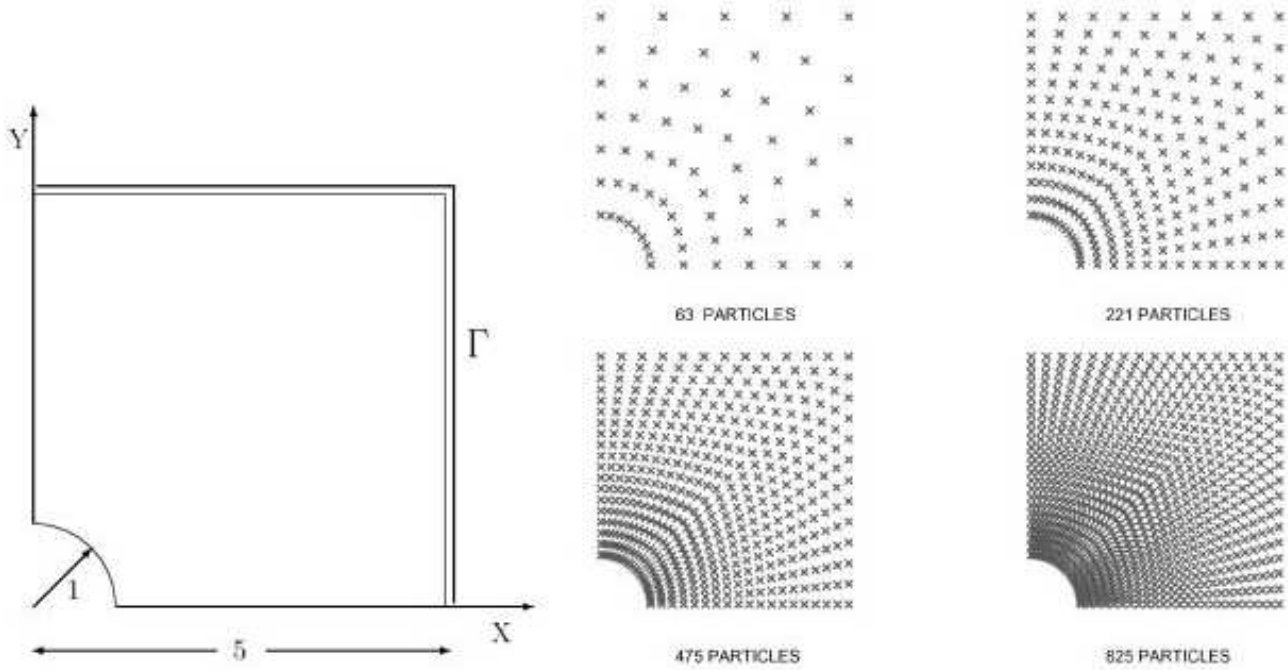

221 PARTICLES
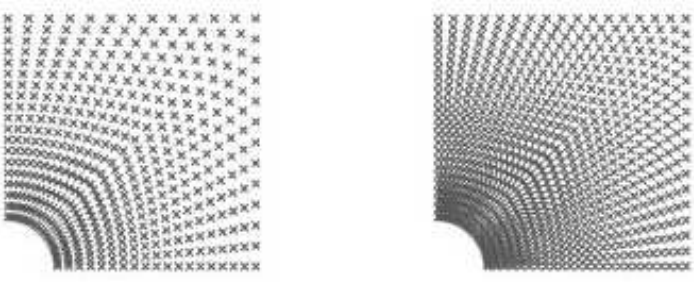

475 PARTICLES

825 PARTICLES

Figure 22. Problem statement for the plate with a hole and discretizations.

\subsection{The plate with a hole}

The stress field of an infinite plate with a hole subject to a far-field unit traction in the $x$ direction is $[22,6]$

$$
\begin{aligned}
& \sigma_{x x}=1-\frac{a^{2}}{r^{2}}\left(\frac{3}{2} \cos (2 \theta)+\cos (4 \theta)\right)+\frac{3 a^{4}}{2 r^{4}} \cos (4 \theta) \\
& \sigma_{y y}=-\frac{a^{2}}{r^{2}}\left(\frac{1}{2} \cos (2 \theta)-\cos (4 \theta)\right)-\frac{3 a^{4}}{2 r^{4}} \cos (4 \theta) \\
& \sigma_{x y}=-\frac{a^{2}}{r^{2}}\left(\frac{1}{2} \sin (2 \theta)+\sin (4 \theta)\right)+\frac{3 a^{4}}{2 r^{4}} \sin (4 \theta)
\end{aligned}
$$

where $a=1$ is the hole radius, $r=\sqrt{x^{2}+y^{2}}$ and $\theta=\arctan (y / x)$. The bounded upper quadrant shown in Figure 22 is used to solve the problem. Symmetry conditions are imposed in $x=0$ and $y=0$ and the tractions of the exact solution are considered in $\Gamma$.

Figure 23 shows the relative energy error with $\mathrm{FE}$ and $\nu=0.3,0.4999$. When $v=0.3$ typical convergence results are obtained with all interpolations. However, all the FE interpolations, even the eight-noded $\mathrm{FE}\left(\mathrm{Q}^{+}{ }^{+}\right)$, suffer from locking when $v=0.4999$. In this example, there is a non-negligible projection of the solution in the space of non-physical locking modes, even for the $2^{+} \mathrm{FE}$. Similar results are obtained with EFG imposing consistency with several polynomial spaces (Figure 24). Two values of $\rho / h$ are considered for the $\mathscr{L}_{1}$ consistency. Again, the behaviour is independent of $\rho / h$. Better results are obtained with large $p / h$ but locking effects are important in all cases. Near the incompressible limit, as in FE, good results cannot be obtained with any of the interpolations. 

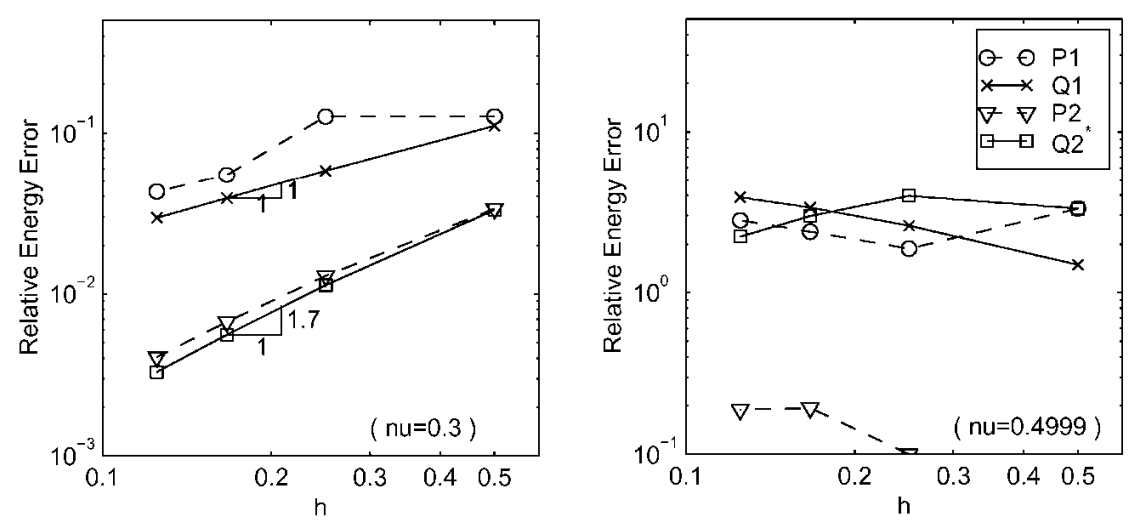

Figure 23. FE, $v=0.3$ (left) and $v=0.4999$ (right).
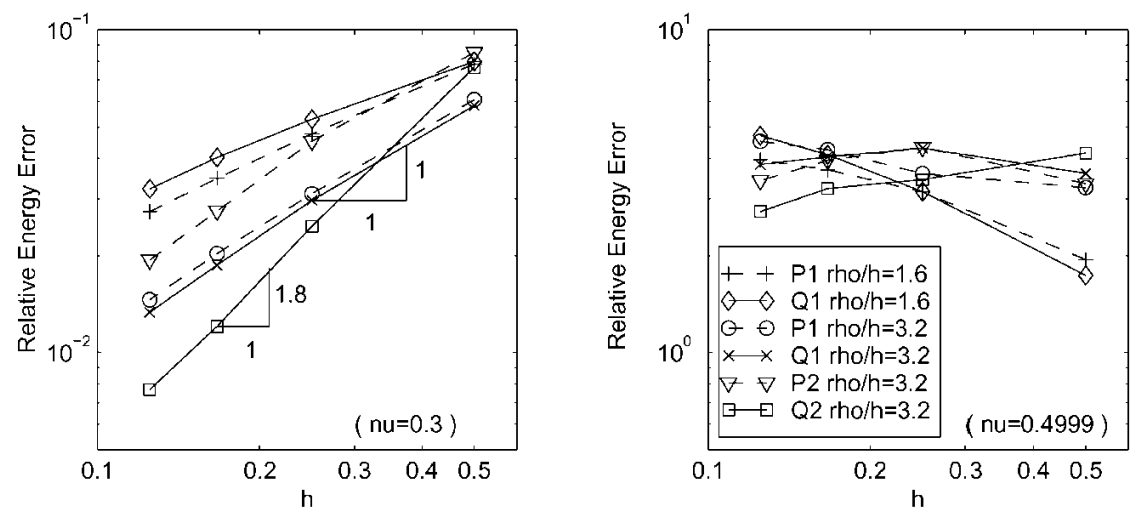

Figure 24. EFG, $v=0.3$ (left) and $v=0.4999$ (right).

\subsection{Prandtl's punch test}

In this section a non-linear example is considered, the so-called Prandtl's punch test [7]. A bilinear perfect elastoplastic material is considered and plane strain conditions are assumed. The domain is shown in Figure 25. It has one axis of symmetry and only a half of the domain is discretized. The prescribed displacements are depicted in the same figure. The cubic spline is used for the weighting functions with rectangular supports. Lagrange multipliers have been used to impose the essential boundary conditions. Figures 26-29 show the evolution of the reaction force against the imposed displacement. The reaction force is normalized by the yield stress $\sigma_{Y}$ and the area.

Figure 26 shows the solution obtained with EFG, imposing consistency in $\mathscr{Q}_{1}$, with a uniform distribution of $9 \times 9$ particles. As noted in Reference [7], locking alleviates as $\rho / h$ increases. Locking effects drastically degrade the solution when $\rho / h=1.2$ (similar to Q1 FE), but a good solution can be obtained with $\rho / h=3.2$. With these results, one could conclude: (1) there are no locking effects when $\rho / h$ is large enough and (2) locking effects decrease as $\rho / h$ increases. Both conclusions would be false. 


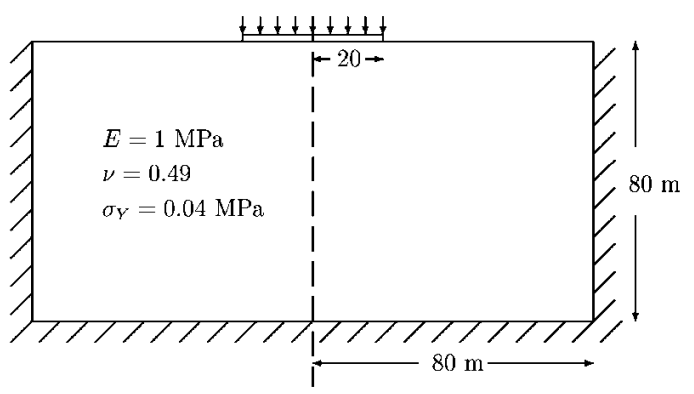

Figure 25. Prandtl's punch test: problem statement.
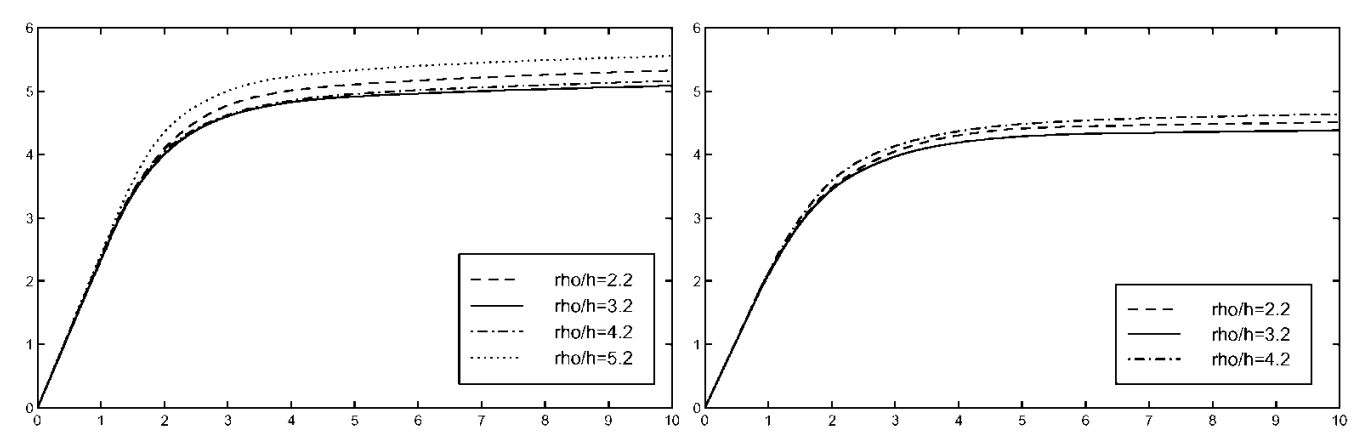

Figure 27. Load versus displacement with $9 \times 9$ (left) and $17 \times 17$ (right) particles, $m=1$ and $v=0.49$.

Figure 27 shows the results with several values of $\rho / h$. The best result is obtained still with $\rho / h=3.2$ but results will degrade if the ratio $\rho / h$ is increased further. Similar results are obtained with a distribution of $17 \times 17$ particles. In both cases there are particles with no interaction with the boundary. Note that the solution is enhanced with the refinement due to the convergence of the interpolation. However, one can observe again an optimal value for the dilation parameter at $\rho / h=3.2$. A summary of these results is shown in Table V. Optimal values for $\rho / h$ are emphasized with bold face.

This is due to the well-known fact that an optimal value of $\rho / h$ exists for a given problem. Here the issue of the optimal ratio of $\rho / h$ is even more crucial because locking clearly influences this optimal value. This is shown in the next case where the same $9 \times 9$ distribution of particles is used and the Poisson coefficient is increased up to $v=0.49999$. Thus, it is false 
INCOMPRESSIBLE LIMIT FOR GALERKIN METHOD

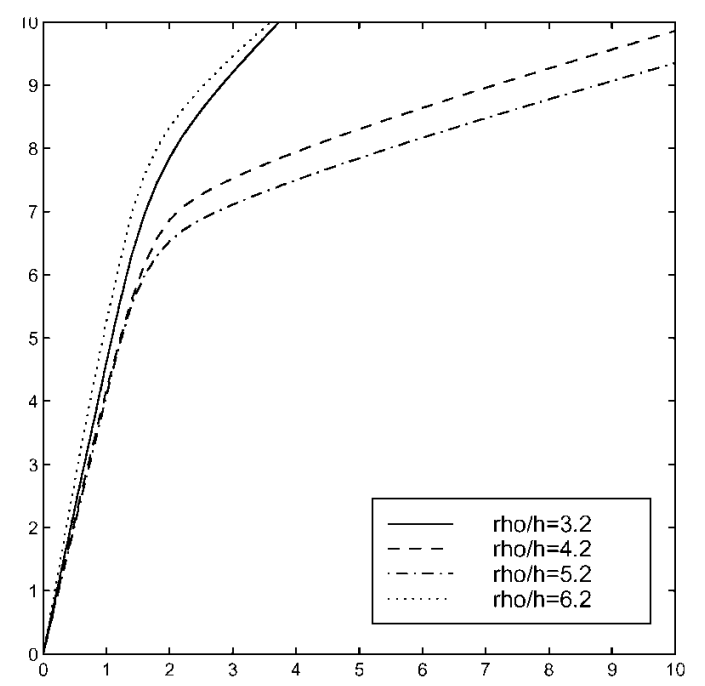

Figure 28. Reaction force versus displacement with $9 \times 9$ particles and $v=0.49999$
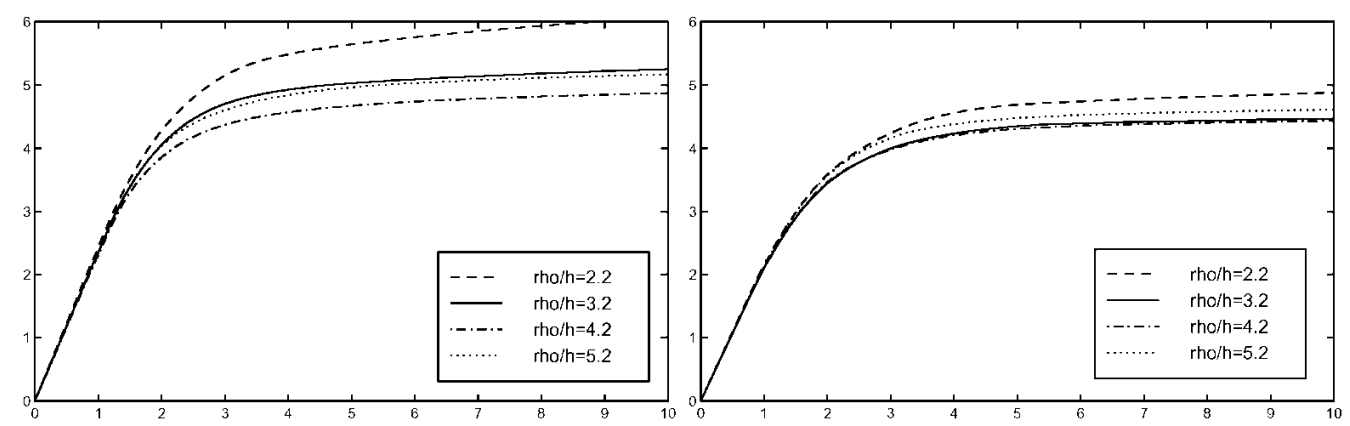

Figure 29. Load versus displacement with $9 \times 9$ (left) and $17 \times 17$ (right) particles, $\mathscr{Q}_{2}$ consistency and $v=0.49$.

Table V. Load at the final displacement for Prandtl's punch test.

\begin{tabular}{lccc}
\hline & $\begin{array}{c}9 \times 9 \text { particles } \\
v=0.49\end{array}$ & $\begin{array}{c}17 \times 17 \text { particles } \\
v=0.49\end{array}$ & $\begin{array}{c}9 \times 9 \text { particles } \\
v=0.49999\end{array}$ \\
\hline$\rho / h=2.2$ & 5.329 & 4.514 & 17.855 \\
$\rho / h=3.2$ & $\mathbf{5 . 0 8 7}$ & $\mathbf{4 . 3 8 5}$ & 16.078 \\
$\rho / h=4.2$ & 5.163 & 4.642 & 9.856 \\
$\rho / h=5.2$ & 5.558 & 4.638 & $\mathbf{9 . 3 4 8}$ \\
$\rho / h=6.2$ & 5.583 & 4.768 & 14.754 \\
\hline
\end{tabular}


that (1) there are no locking effects when $\rho / h$ is large enough and (2) locking effects decrease as $\rho / h$ increases.

Table $\mathrm{V}$ also includes the results obtained with a Poisson coefficient $y=0.49999$. The evolution of the reaction force versus the displacement close to the incompressible limit are shown in Figure 28. Now the optimal value for the dilation parameter is no longer $\rho / h=3.2$ but it is in the neighbourhood of $\rho / h=5.2$. Nevertheless, the solution is excessively bad because of the locking effects for all values of $\rho$. The discretization must be refined in order to obtain an acceptable solution.

That is, an increase in the dilation parameter $\rho$ can alleviate locking. However, it is not advisable to arbitrarily increase the dilation parameter. An increase in the ratio $\rho / h$ will induce a larger band-width with its corresponding increase in the computational cost. Moreover, in the examples, there is an optimal value for $\rho / h$ that gives the best result: the solution degrades when $\rho / h$ is larger than this optimal value. However, even at this optimal value the solution can be useless because of locking effects.

On the other hand, in this example, an increase in the order of consistency does not sufficiently improve the solution. The non-physical locking modes for the $\mathscr{Q}_{2}$ consistency have an important component in the solution. Figure 29 shows the reaction force against the displacement with $y=0.49$ and several values of $\rho / h$. Again there is an optimal value for the ratio $\rho / h$, now in the neighbourhood of $\rho / h=4.2$.

\section{CONCLUSIONS}

A modal analysis has been used to study volumetric locking in EFG. The number of nonphysical locking modes has been compared with equivalent finite element formulations. The influence of the ratio between the dilation parameter and the distance between particles, $\rho / h$, has been studied independent of the consistency required. Finally, some numerical examples have been used to corroborate the results of the modal analysis. The major conclusions are:

1. The number of non-physical locking modes is independent of the ratio $\rho / h$.

2. An increase of the dilation parameter decreases the eigenvalue (amount of energy) of the locking mode attenuating, but not suppressing, the volumetric locking (in the incompressible limit the same problems will occur).

3. An increase in the order of consistency decreases the number of non-physical locking modes.

4. The decrease in the number of non-physical locking modes is slower than in finite elements. Thus EFG will not improve the properties of the FEM (from a volumetric locking viewpoint) when $p$ or $h p$ refinement is enforced. However, for practical purposes and as in finite elements, in EFG an $h p$ strategy will also suppress locking. Moreover, note that EFG incorporates an $h p$ strategy in a simpler and more natural manner.

\section{REFERENCES}

1. Hughes TJR. The Finite Element Method. Prentice-Hall: Englewood Cliffs, NJ, 1987.

2. Armero F. On the locking of standard finite elements. Classroom Notes. University of California, Berkeley, 1999.

3. Suri M. Analytical and computational assessment of locking in the hp finite element method. Computer Methods in Applied Mechanics and Engineering 1996; 133:347-371. 


\section{INCOMPRESSIBLE LIMIT FOR GALERKIN METHOD}

4. Belytschko T, Lu YY, Gu L. Element-free Galerkin methods. International Journal for Nunerical Methods in Engineering 1994; 37:229-256.

5. Zhu T, Atluri SN. A modified collocation method and a penalty formulation for enforcing the essential boundary conditions in the element free Galerkin method. Computational Mechanics 1998; 21:211-222.

6. Dolbow J, Belytschko T. Volumetric locking in the element free Galerkin method. International Joumal for Numerical Methods in Engineering 1999; 46:925-942.

7. Askes H, de Borst R, Heeres 0 . Conditions for locking-free elasto-plastic analyses in the element-free Galerkin method. Computer Methods in Applied Mechanics and Engineering 1999; 173:99-109.

8. Chen J, Yoon S, Wang H, Liu WK. An improved reproducing kernel particle method for nearly incompressible finite elasticity. Computer Methods in Applied Mechanics and Engineering 2000; 181:117-145.

9. Belytschko T, Krongauz Y, Organ D, Fleming M, Krysl P. Meshless methods: an overview and recent developments. Computer Methods in Applied Mechanics and Engineering 1996; 139:3-47.

10. Liu WK, Belytschko T, Oden JT (eds). Meshless methods. Computer Methods in Applied Mechanics and Engineering 1996; 139:1-440.

11. Liu WK, Chen Y, Jun S, Chen JS, Belytschko T, Pan C, Uras RA, Chang CT. Overview and applications of the reproducing kernel particle methods. Archives of Computational Methods in Engineering, State of the Art Reviews 1996; 3:3-80.

12. Liu WK, Li S, Belytschko T. Moving least square reproducing kernel methods. (I) Methodology and convergence. Computer Methods in Applied Mechanics and Engineering 1997; 143:113-154.

13. Nayroles B, Touzot G, Villon P. Generating the finite element method: diffuse approximation and diffuse elements. Computational Mechanics 1992; 10:307-318.

14. Liu WK, Jun S, Zhang YF. Reproducing kernel particle methods. International Joumal for Numerical Methods in Fluids 1995; 20:1081-1106.

15. Liu WK, Chen Y. Wavelet and multiple scale reproducing kernel methods. International Joumal for Numerical Methods in Fluids 1995; 21:901-931.

16. Belytschko T, Organ D, Krongauz Y. A coupled finite element-free Galerkin method. Computational Mechanics $1995 ; 17: 186-195$.

17. Belytschko T, Organ D. Element-free Galerkin methods for dynamic fracture in concrete. In Comp. Plasticity. Fundamentals and Applications, Owen DRJ, Oñate E, Hilton E (eds). CIMNE, Barcelona, 1997; 304-321.

18. Belytschko T, Tabbara M. Dynamic fracture using element-free Galerkin methods. International Journal for Numerical Methods in Engineering 1996; 39:923-938.

19. Lu YY, Belytschko T, Gu L. A new implementation of the element free Galerkin method. Computer Methods in Applied Mechanics and Engineering 1994; 113:397-414.

20. Organ D, Fleming M, Terry T, Belytschko T. Continuous meshless approximations for nonconvex bodies by diffraction and transparency. Computational Mechanics 1996; 18:225-235.

21. Huerta A, Fernández-Méndez S. Enrichment and coupling of the finite element and meshless methods. International Joumal for Numerical Methods in Engineering 2000; 48:1615-1636.

22. Timoshenko SP, Goodier JN. Theory of Elasticity (3rd edn). McGraw-Hill: New York, 1987. 